\title{
High-resolution radio emission from RCW 49/Westerlund 2
}

\author{
P. Benaglia ${ }^{1,2, \star}$, B. Koribalski ${ }^{3}$, C. S. Peri ${ }^{1,2}$, J. Martí ${ }^{4}$, J. R. Sánchez-Sutil ${ }^{4}$, S. M. Dougherty ${ }^{5}$, and A. Noriega-Crespo ${ }^{6}$ \\ 1 Instituto Argentino de Radioastronomía, CCT-La Plata (CONICET), C.C.5, 1894 Villa Elisa, Argentina \\ e-mail: pbenaglia@fcaglp.unlp.edu.ar \\ 2 Facultad de Ciencias Astronómicas y Geofísicas, UNLP, Paseo del Bosque s/n, 1900 La Plata, Argentina \\ 3 Australia Telescope National Facility, CSIRO Astronomy \& Space Science, PO Box 76, NSW 1710 Epping, Australia \\ ${ }^{4}$ Escuela Politécnica Superior de Jaén, Universidad de Jaén, Campus Las Lagunillas, Edif. A3, 23071 Jaén, Spain \\ e-mail: [jmarti;jrssutil]@ujaen.es \\ 5 National Research Council of Canada, Herzberg Institute for Astrophysics, Dominion Radio Astrophysical Observatory, \\ PO Box 248, Penticton, BC V2A 6J9, Canada \\ ${ }^{6}$ Infrared Processing and Analysis Center, California Institute of Technology, Pasadena, CA 91125, USA
}

Received 27 May 2013 / Accepted 14 August 2013

\section{ABSTRACT}

\begin{abstract}
Aims. The HII region RCW 49 and its ionizing cluster form an extensive, complex region that has been widely studied at infrared (IR) and optical wavelengths. The Molonglo $843 \mathrm{MHz}$ and Australia Telescope Compact Array data at 1.4 and $2.4 \mathrm{GHz}$ showed two shells. Recent high-resolution IR imaging revealed a complex dust structure and ongoing star formation. New high-bandwidth and high-resolution data of the RCW 49 field have been obtained to survey the radio emission at arcsec scale and investigate the smallscale features and nature of the HII region.

Methods. Radio observations were collected with the new 2-GHz bandwidth receivers and the CABB correlator of the Australia Telescope Compact Array [ATCA], at 5.5 and $9.0 \mathrm{GHz}$. In addition, archival observations at 1.4 and $2.4 \mathrm{GHz}$ have been re-reduced and re-analyzed in conjunction with observations in the optical, IR, X-ray, and gamma-ray regimes.

Results. The new 2-GHz bandwidth data result in the most detailed radio continuum images of RCW 49 to date. The radio emission closely mimics the near-IR emission observed by Spitzer, showing pillars and filaments. The brightest continuum emission comes from the region known as the bridge. The overall flattish spectral index is typically consistent with a free-free emission mechanism. However, hints of nonthermal components are also present in the bridge. An interesting jet-like structure surrounded by a bubble feature whose nature is still unclear has been discovered close to the Westerlund 2 core. Two apparent bow shocks and a number of discrete sources have been detected as well in the surroundings of RCW 49. In addition, we also report on and discuss the possible detection of a hydrogen recombination line.

Conclusions. The radio results support an association between the $\mathrm{cm}$ continuum and molecular emission. The detection of the radio recombination line kinematically favors a RCW 49 distance of 6-7 kpc. If the negative spectral indices measured at the bridge should be confirmed to be caused by synchrotron emission, we propose a scenario where high-energy emission could be produced. Finally, the newly discovered jet-like structure appears to be an intriguing source that deserves a detailed study by itself.
\end{abstract}

Key words. stars: winds, outflows - ISM: individual objects: RCW 49 - open clusters and associations: individual: Westerlund 2 radio continuum: ISM - gamma rays: stars

\section{Introduction}

RCW 49 has been one of the most often studied HII regions of the southern sky since its discovery in the sixties (Rodgers et al. 1960) in $\mathrm{H} \alpha$ emission. As part of the comprehensive study of more than $200 \mathrm{HII}$ regions, Goss \& Shaver (1970) were the first to characterize and image the source at $5 \mathrm{GHz}$. The region was extensively studied in radio recombination lines with single-dish instruments (see Caswell \& Haynes 1987, and references therein). Until now, the low declination of RCW 49, which makes it inaccessible for northern interferometers, combined with the very large angular extent $\left(\sim 40^{\prime} \times 50^{\prime}\right)$ which made observing excessively time-consuming, precluded arcsec resolution $\mathrm{cm}$-radio observations.

Images obtained at IR wavelengths (Whitney et al. 2004) in the area of the Spitzer-GLIMPSE program (Benjamin et al. 2003) pointed to the cluster Westerlund $2[\mathrm{Wd} 2]$ as the

\footnotetext{
* Member of Conicet.
}

ionizing agent. This cluster is among the five super star clusters known in the galaxy (Johnson 2005), with stellar densities above $10^{4}$ stars per $\mathrm{pc}^{3}$. Piatti et al. (1998) derived an age of 2-3 Myr and an average visual absorption of $5 \mathrm{mag}$. Ascenso et al. (2007) estimated a total stellar mass up to $\sim 7 \times 10^{4} M_{\odot}$, assuming a distance of $2.8 \mathrm{kpc}$. Over five decades, distance values derived for both the HII region and the super star cluster presented huge scatter, ranging from 2.5 to $8 \mathrm{kpc}$. The distance to the region remains an open topic.

A $\sim 15^{\prime \prime}$ by $15^{\prime \prime}$ field centered on Westerlund 2 was surveyed in X-rays by Tsujimoto et al. (2007) and Nazé et al. (2008) with Chandra. Tsujimoto and collaborators detected X-ray emission from the early-type stars, cataloged hundreds of cluster members, mostly pre-main-sequence and early-type stars, and identified about 30 new OB star candidates. This led them to derive a constraint of 2-5 kpc for the distance to the cluster, based on the mean luminosity of T Tauri stars. Nazé et al. (2008) measured and explained the brightness variability of the eclipsing, colliding-wind binary WR 20a and found that faint, soft and 
diffuse emission pervades the field of view, but no clear structure could be identified. Fujita et al. (2009) analyzed Suzaku observations of Westerlund 2 and found diffuse X-ray emission consisting of thermal and maybe one nonthermal component ${ }^{1}$.

The extended TeV source HESS J1023-575 is positionally coincident with the complex RCW 49/Westerlund 2 (Aharonian et al. 2007) and constitutes evidence for the presence of relativistic particles in the field. Follow-up H.E.S.S. observations (Abramowski et al. 2011) still did not determine the nature of the $\mathrm{TeV}$ emission. Fermi observations resolved the GeV emission into two different sources: 2FGL J1022.7-5741 and 2FGL J1023.5-5749c (Nolan et al. 2012). The first of these two sources is now believed to be related to the pulsar PSR J1022-5746 (Dormody et al. 2009), while the second one is not yet clearly associated with any known source (see Fig. 1). One potential source of high-energy photons is relativistic particles that may be evident in radio observations. However, no clear connection between the radio emission in the region and the high-energy sources has yet been established.

The latest comprehensive study of RCW 49 at radio frequencies was published by Whiteoak \& Uchida (1997) [WU97]. The authors observed the source with the Molonglo Observatory Synthesis Telescope (MOST) at $843 \mathrm{MHz}$ and with the Australia Telescope Compact Array (ATCA) ${ }^{2}$ at 1.4 and $2.4 \mathrm{GHz}$, attaining angular resolutions of 44" to $7^{\prime \prime}$. WU97 described the detected emission as two ring-like features or shells (see Fig. 2). The authors proposed that the northern one, with a blister toward the west, was created by the rich cluster Westerlund 2 and that the wind of the well-known WR star in the field, WR 20b, could have built the southern ring. The area where the two shells seem to overlap shows the brightest radio emission, and is identified here as the bridge.

Molecular lines have been studied in the region by several authors (Ohama et al. 2010, and references therein) at arcmin angular resolution. For instance, Furukawa et al. (2009) pointed out that several clouds exist with local standard of rest (LSR) velocities between -11 and +9 and between 11 and $21 \mathrm{~km} \mathrm{~s}^{-1}$.

Spitzer IRAC observations of RCW 49 showed that dust coexists with ionized gas, or is embedded in neutral gas mixed with ionized gas (Churchwell et al. 2004). The data also let Whitney et al. (2004) conclude that star formation is occuring at present, continuous and/or triggered by stellar winds and shocks of the Westerlund 2 stellar population. Deep cm-wavelength radio images at a similar angular resolution are needed to clarify the association between dust and ionized matter. They could also pinpoint protostellar objects and star-forming regions.

Very recently, the capabilities of the main radio interferometers have been considerably extended. The receiver bandwiths, inversely proportional to the square root of the attainable noise, have been enlarged by a factor of ten and more. We have obtained high-sensitivity arcsec resolution radio observations using the upgraded facilities to search for point-source emission and extended low surface brightness emission from the entire RCW 49 field and surroundings. We aim to look for a correlation between the radio, IR, optical, and high-energy emission in the field and study the radiation regime.

The content of this paper is as follows. Section 2 describes the new broad-bandwidth observations and the reduction

\footnotetext{
1 We used the convention $S_{v} \propto v^{\alpha}$, where $S_{v}$ is the flux density at a frequency $v$ and $\alpha$ is the spectral index.

2 The Australia Telescope Compact Array is funded by the Commonwealth of Australia for operation as a National Facility by CSIRO.
}

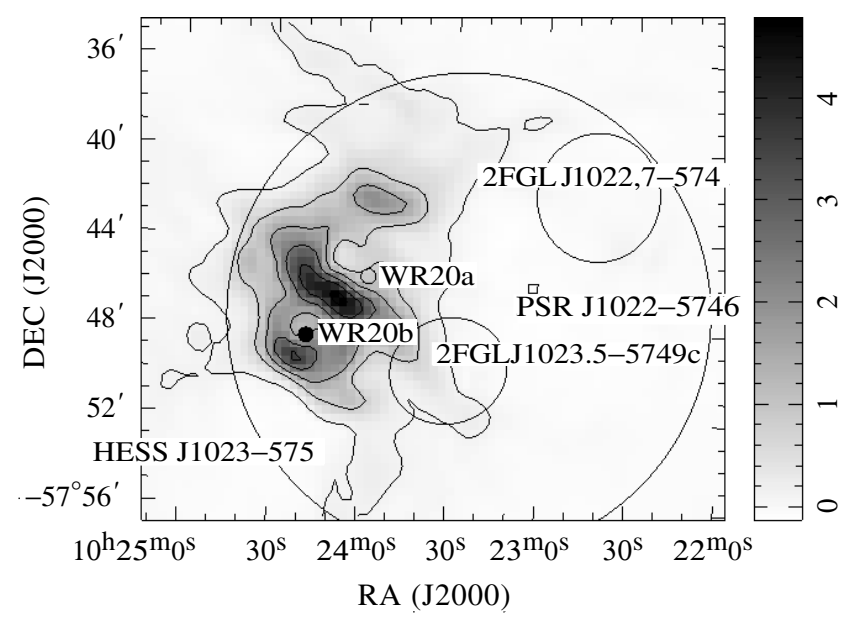

Fig. 1. Field of RCW 49. Emission at $843 \mathrm{MHz}$ (Whiteoak \& Uchida 1997 ) is presented in contours and grayscale (in units of Jy). The positions of the two very bright stars WR 20a (member of Westerlund 2) and WR 20b and of the high-energy sources 2FGLJ 1022-5741.7, 2FGLJ 1023.5-5749c, HESS J1023-575, and PSR J1022-5746 are shown.

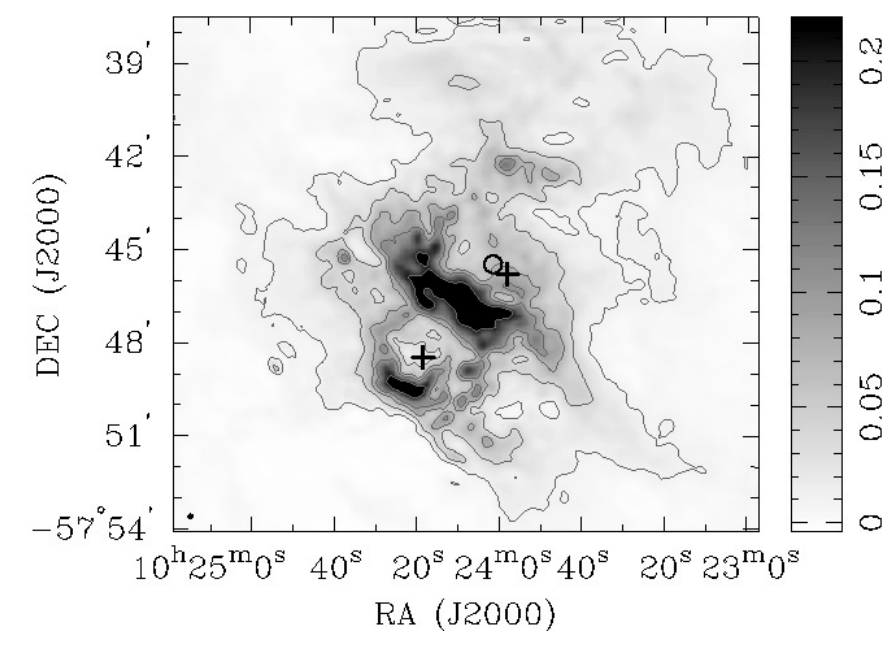

Fig. 2. Image of the reprocessed 1.4-GHz data taken by WU97 of RCW 49 (see Sect. 2.3). The position of Westerlund 2 is marked by the circle. The two very bright stars WR 20a (member of Westerlund 2) and WR $20 \mathrm{~b}$ are indicated by the two crosses. The bridge is clearly seen as the bright bar of emission in the center of the image to the southeast of Westerlund 2. Farther to the southeast is WR20b, surrounded by a circle of emission, the southern ring as identified by WU97. There is a suggestion of the northern ring around Westerlund 2, but it is not as clear as the one to the south, mainly because the western side is absent as a result of the "blister" suggested by WU97.

process. In Sect. 3 we report the data analysis, including an analysis of the spectral index. Section 4 presents additional findings on correlations with emission at other frequency ranges. Section 5 contains a discussion of the distance to the complex and the putative relation between the radio emission and the high-energy sources. Section 6 presents our conclusions and ideas for future work.

\section{Radio observations and data reduction}

\subsection{Continuum data}

Radio observations toward RCW 49 were carried out with the Australia Telescope Compact Array (ATCA, project C1847) 
Table 1. Main parameters of the CABB ATCA images at 5.5 and $9 \mathrm{GHz}$.

\begin{tabular}{lcc}
\hline \hline Parameter & $5.5-\mathrm{GHz}$ image & $9.0-\mathrm{GHz}$ image \\
\hline Synthesized beam & $3^{\prime \prime} \times 3^{\prime \prime}$ & $2^{\prime \prime} \times 2^{\prime \prime}$ \\
rms $\left(\mathrm{mJy}\right.$ beam $\left.^{-1}\right)$ & 0.6 & 0.6 \\
Min/Max $\left(\mathrm{mJy} \mathrm{beam}^{-1}\right)$ & $-1.3 / 22.6$ & $-1.4 / 55.9$ \\
Flux density $(\mathrm{Jy})$ & $146 \pm 12$ & $103 \pm 30$ \\
Baseline uv range $(\mathrm{k} \lambda)$ & $0.5-129$ & $0.8-198$ \\
\hline
\end{tabular}

Notes. ${ }^{(\dagger)}$ Total flux density measured above $5 \sigma(=5 \mathrm{rms})$ over the RCW 49 region.

using the Compact Array Broadband Backend (CABB, Wilson et al. 2011). Two array configurations were used, 6A on 2012 February 20 and 750D on 2012 February 22, for $12 \mathrm{~h}$ each. Observations at $5.5 \mathrm{GHz}$ ( $C$ band) and $9.0 \mathrm{GHz}$ ( $X$ band) were obtained simultaneously, each with a bandwidth of $2 \mathrm{GHz}$. They were planned to derive information on the spectral index of the radiation. Hence, 5.5 and $9.0 \mathrm{GHz}$ were chosen for their high resolution (relative to 1.4 and $2.4 \mathrm{GHz}$ ) and as frequencies where any potential nonthermal emission can still be detected. With the combination of configurations and frequencies mentioned above we expected to be able to image structures between 1 and $100^{\prime \prime}$ in extent.

The observed field comprised the extension of the $\mathrm{TeV}$ source HESS J1023-575 and the cm-radio emission measured by WU97, covering an area of $\sim 30 \mathrm{arcmin}^{2}$. The observing strategy consisted of building a mosaic with 41 pointings, ensuring Nyquist sampling at the higher frequency band. The integration time for each pointing was approximately $13 \mathrm{~min}$. The bright source PMN J1047-6217 was used to calibrate the antenna gain phase, and monitored before and after each mosaic observation so that the gain phase could be interpolated through the mosaic observations. The absolute flux scale was determined from PKS 1934-638, assuming flux density values of 5.1 Jy $(5.5 \mathrm{GHz})$ and $2.74 \mathrm{Jy}(9.0 \mathrm{GHz})$.

The multichannel image reconstruction, image analysis and display (MIRIAD) routines ${ }^{3}$ (Sault et al. 1995) were used to perform the data editing, calibration and image reconstruction. A number of factors complicated the reduction and imaging process, namely: (i) the large angular extension of the radio source, which required mosaicing; (ii) the complex nature of the radio source with a highly dynamical range of the emission comprising bright ridges, faint extensions, loops, and shells; (iii) the broad $\mathrm{CABB}$ bandwidth of $2-\mathrm{GHz}$ presents a high fractional bandwidth and correspondingly wide ranges in primary and synthesized beams, plus variations in the emission level across the band due to spectral index variations; and (iv) the ability of MIRIAD tasks to deal with these demanding observations and large data sets.

Various approaches were tested to construct the images using the tasks MOSSDI, MOSMEM, and MFCLEAN. It was found that the maximum entropy deconvolution MOSMEM was the best tool not only for minimizing side lobes, but for dealing with different levels of radio luminosity from the various pointings. Results from the CLEAN algorithm were also examined, but remained less satisfactory, as expected for extended low-surface brightness emission when compared with maximum entropy methods. A similar rms noise level of $\leq 0.6 \mathrm{mJy} \mathrm{beam}^{-1}$ was attained at both frequency bands, as determined from outer regions of the field where no emission from RCW 49 was evident. This

\footnotetext{
3 http://www.atnf.csiro.au/computing/software/miriad/
}

noise level is equivalent to $3 \times$ and $2 \times$ the theoretical rms values at 5.5 and $9 \mathrm{GHz}$, respectively. The resulting images at the two frequencies are shown in Fig. 3.

Goss \& Shaver (1970) imaged the RCW 49 complex with the Parkes radiotelescope at $5000 \mathrm{MHz}$ (FWHM: 4.1', see their Fig. 6). The authors detected the source G284.3-0.3, positionally coincident with RCW 49, as well as a much fainter source to the east (G284.6-0.2), and quoted an integrated flux for both sources of $335 \mathrm{Jy}$. Later on, Churchwell et al. (1974) observed the region in continuum and line emission. They described the region as an area of $5^{\prime} \times 7^{\prime}$ and derived an integrated flux density of $178.8 \mathrm{Jy}$ at $5 \mathrm{GHz}$. The integrated flux at $5.5 \mathrm{GHz}$ for the data presented here, above $5 \sigma$, is given in Table 1 , and above $3 \sigma$ is $\sim 160 \pm 15 \mathrm{Jy}$. A comparison between this last flux density value and that published by Churchwell et al. (1974) shows that the interferometric data gather about $90 \%$ of the flux measured by a single-dish telescope.

\subsection{Line data}

We took advantage of the CABB-ATCA simultaneous zoom modes and performed a pilot line observation with the 750-D configuration. The central frequency was $5005 \mathrm{MHz}$, at which two transitions are expected: the $\mathrm{H} 137 \beta$ radio recombination line and the $3{ }_{1}-3_{1}$ A-branch line of methanol (Robinson et al. 1974). We set the zoom modes of the correlator to cover 2560 channels of $0.5 \mathrm{kHz}$ width each. This corresponds to a velocity resolution of $0.05 \mathrm{~km} \mathrm{~s}^{-1}$. The final line-only data cube was built with robust weighting set to zero, applying hanning smoothing to reduce the number of channels to 270 , each of $0.5 \mathrm{~km} \mathrm{~s}^{-1}$ width.

\subsection{Archive 1.4 and $2.4 \mathrm{GHz}$ data}

The archive 1.4 and $2.4 \mathrm{GHz}$ image data sets from the WU97 project, campaign C492, were re-reduced and imaged. The project consisted of a 12-h observation at $1.5 \mathrm{C}$ array and a 12 -h observation at the $1.5 \mathrm{~B}$ array, with central frequencies of 1.376 and $2.378 \mathrm{MHz}, 128 \mathrm{MHz}$ bandwidth each. As with the new observations presented in this paper, the archival data were edited and calibrated with the MIRIAD package. Images were built using maximum-entropy algorithms with a synthesized beam of 10 arcsec. The resulting image rms at $1.376 \mathrm{GHz}$ was $3 \mathrm{mJy} /$ beam, and at $2.378 \mathrm{GHz}$ it was $1 \mathrm{mJy} /$ beam.

\section{Analysis of the radio data}

The ATCA 5.5 and $9 \mathrm{GHz}$ continuum images (Fig. 3) show a high degree of detail compared with the observations of WU97 (their Fig. 3, and Fig. 2 presented here), which were described by the northern and southern shells, separated by the bridge. Figure 3 confirms this simple picture for the largest-scale emission in RCW 49, but at the highest resolution the new data reveal much more complex structures. At both frequencies, complexes of pillars or fingers of emission are very clear in the bridge. A plethora of filaments of different sizes, widths, and intensities dominate the emission. Clouds and circular-elliptical sources of a few seconds of arc are also visible. Though somewhat less evident, there is at least one bow-shock-like feature at RA, Dec $(\mathrm{J} 2000)=10: 24: 38.73,-57: 45: 19.9$, marked in Fig. 4 as $\mathrm{S} 3$.

There are emission minima in the vicinity of the stars WR 20a and WR 20b. The average minimum flux density in the region of Westerlund 2 is $\sim 1$ mJybeam $^{-1}$ at $5.5 \mathrm{GHz}$, 

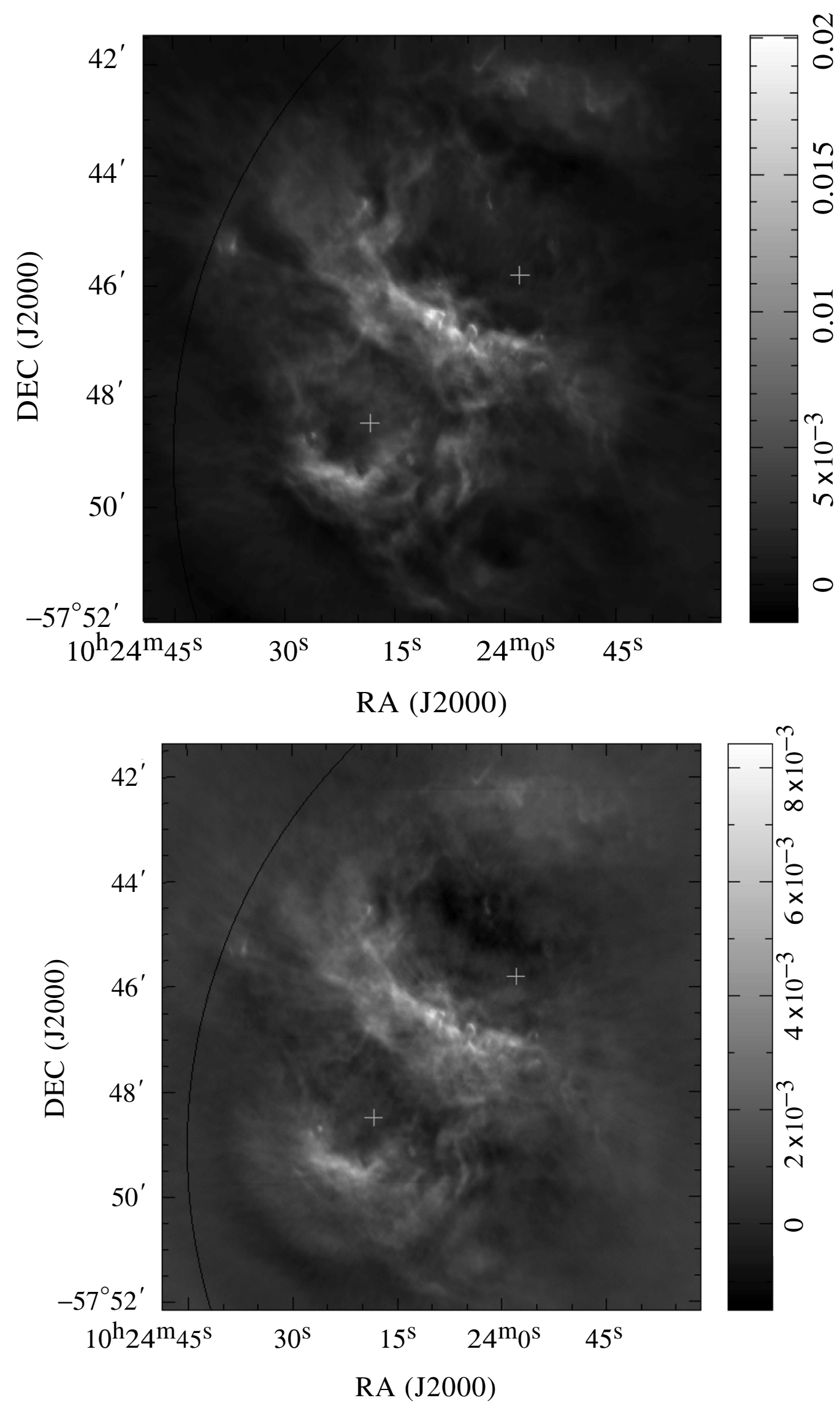

Fig. 3. Top: the 5.5-GHz ATCA image of RCW 49 field. The synthesized beam is $1.9^{\prime \prime} \times 1.5^{\prime \prime}$. Bottom: the 9.0-GHz image of the RCW 49 field. The synthesized beam is $1.2^{\prime \prime} \times 0.9^{\prime \prime}$. The brightness level in Jy beam ${ }^{-1}$ is shown to the right of each image. The position of the stars WR 20a (N) and WR $20 \mathrm{~b}(\mathrm{~S})$ is indicated with light-gray crosses. The dark-gray circular sector represents the angular size of the extended TeV source HESS J1023-575. 


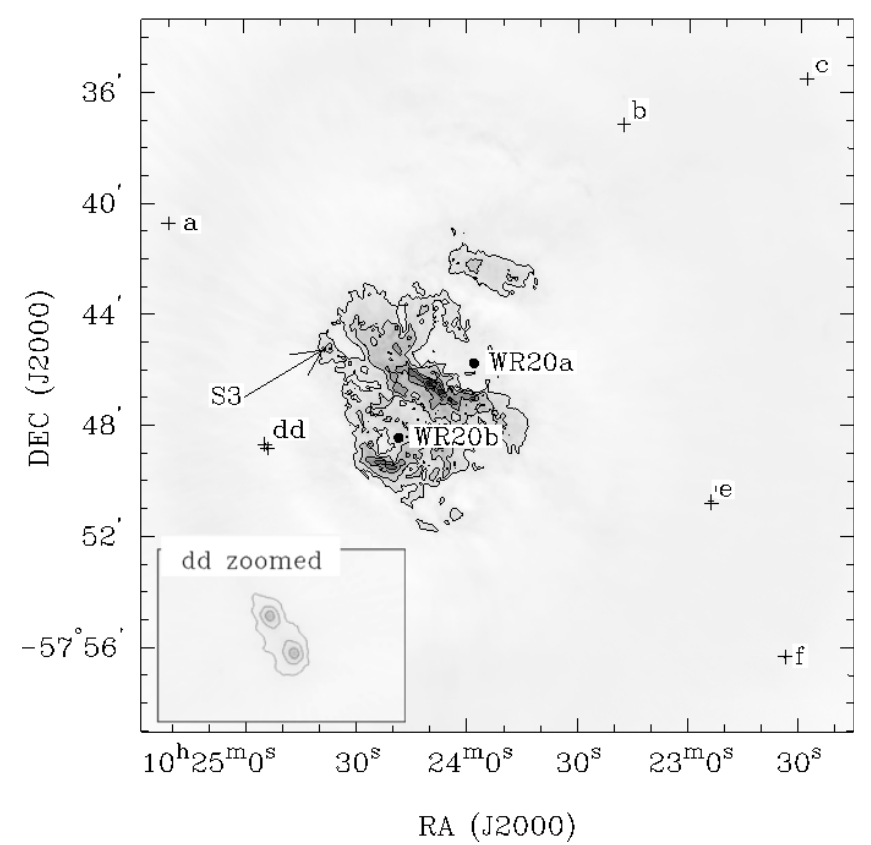

Fig. 4. ATCA $5.5 \mathrm{GHz}$ image of RCW 49 and surroundings. The contour levels are $-0.6,3,9,20,35$, and $60 \mathrm{mJy}$ beam $^{-1}$; the synthesized beam is $3^{\prime \prime} \times 3^{\prime \prime}$. Crosses mark the positions of radio sources detected near RCW 49; these are cataloged in Table 2. The arrow shows the position of a bow-shaped structure, S3 (see Sect. 3.4). The WR stars 20a and $20 \mathrm{~b}$ are marked with solid circles. The inset shows the emission of source "dd", with contour levels of 2, 4, and 8 mJy beam $^{-1}$.

and $\sim-0.5 \mathrm{mJybeam}^{-1}$ at $9 \mathrm{GHz}$. The corresponding one in the region of WR $20 \mathrm{~b}$ is $\sim 0.7 \mathrm{mJy}^{-1}$ aeam ${ }^{-1}$ at $5.5 \mathrm{GHz}$, and $\sim 0.3 \mathrm{mJy}^{-1}$ beam ${ }^{-1}$ at $9 \mathrm{GHz}$.

The observed field contains some of the brightest massive, early-type stars in the Galaxy. No radio emission from the direction of the 3.7-day period massive binary WR 20a, WR 20b or the Westerlund 2 core is detected above 5- $\sigma$ level in the images shown in Fig. 3. A search of an image generated only with high-spatial frequency data, that is, long baselines, to reduce the impact of the large-scale diffuse emission also failed to detect any point source emission in these directions. We do not expect to detect thermal wind sources. For a canonical WR star with a mass-loss rate of $\sim 3 \times 10^{-5} M_{\odot} \mathrm{yr}^{-1}$ (e.g. Chapman et al. 1999) and wind speed $\sim 1000 \mathrm{~km} \mathrm{~s}^{-1}$, the expected thermal flux is $0.3 \mathrm{mJy}$ for a distance of $4 \mathrm{kpc}$.

\subsection{Discrete sources in the surroundings of RCW 49}

We identified six discrete isolated radio sources in the $5.5-\mathrm{GHz}$ continuum image in the field surrounding RCW 49 labeled "a", "b", "c", "dd" (double), "e", and "f" (see Fig. 4).

In Table 2 we list the results of Gaussian fits to the discrete sources: peak coordinates (J2000), total flux density, and peak flux density. The Simbad and NED databases were searched for counterparts at these positions. We also searched the DSS2 and Spitzer-GLIMPSE images. The results of the search are also listed in Table 2, where $D$ is the angular distance between the discrete source and the nearest cataloged object. Given the separations of potential counterparts from the radio source positions relative to the 2 -arcsec beam of the radio data, we conclude that we only detected radio emission from one previously identified source, dd 1 .
The double source dd, composed of dd1 and dd2, was also detected at $9.0 \mathrm{GHz}$. The proposed counterpart, positionally coincident with dd1, was identified by Tsujimoto et al. (2007) as a young stellar object (YSO) candidate. Both radio components are discrete sources at 5.5 and $9 \mathrm{GHz}$ resolution, with total flux densities at $9.0 \mathrm{GHz}$ of $S(\mathrm{dd} 1)=12.2 \mathrm{mJy}$ and $S(\mathrm{dd} 2)=12.1 \mathrm{mJy}$. The fitted $\mathrm{rms}$ at $9.0 \mathrm{GHz}$ is $0.1 \mathrm{mJy}$ beam $^{-1}$. The spectral index between 5.5 and $9 \mathrm{GHz}$ of each component is $\alpha(\mathrm{dd} 1) \sim-0.9$ and $\alpha(\mathrm{dd} 2) \sim-0.8$. Only the component dd 1 appears to correspond with the position of the YSO candidate. However, taking into account the morphology and the spectral indices, we propose that the source dd probably has an extragalactic origin ${ }^{4}$.

\subsection{Spectral index analysis}

Spectral index maps were generated between pairs of observation data sets taken simultaneously with the ATCA.

For each set of data in the analysis, we prepared the data at the two frequencies in the following way: we considered visibilities from baselines sensitive to emission at the same angular scales, that is, we limited the $u v$ range to the same lower limit at both frequencies. We also convolved the higher-frequency map with a synthesized beam of the same size as that of the lower frequency data. In assembling the spectral index maps and spectral index error maps we used only pixels with a signal-to-noise ratio higher than 5 . It should be noted that this analysis does not include the flux on the largest scales, that is, at the lowest spatial frequencies, since they are not sampled at both observing frequencies. Thus, the spectral index information must be interpreted with some caution.

Map between 1.4 and $2.4 \mathrm{GHz}$ pre-CABB ATCA data. The data sets at 1.4 and $2.4 \mathrm{GHz}$ (project C492, Whiteoak \& Uchida 1997) comprised observations performed simultaneously at the two bands. We restricted the generation of the images to visibilities with a minimum $u v$ range of $0.25 \mathrm{k} \lambda$. Figure 5 presents the resulting spectral index map, along with the uncertainty map.

The majority of the region where the $1.4-\mathrm{GHz}$ emission is brightest (the bridge) has spectral index values $\sim 0$, with uncertainties of less than 0.2 . We interpret this as optically thin thermal emission that we expect in a large diffuse HII region such as RCW 49. However, we also see some evidence in the bridge of spectral index values that are steeper than -0.4 , with relatively low uncertainties. Of most interest are the areas steeper than -0.4 that are in the areas with some of the brightest 2.4-GHz emission. Outside these bright regions, the indication of steep indices is less robust since we may be missing some emission at the higher frequency on the larger scales, as inferred by the steepest indices appearing where the high frequency emission level is low, that is, on the northern edge of the bridge and the southern edge of the south shell.

Map between 5.5 and $9.0 \mathrm{GHz} C A B B$ data. In configurations $6 \mathrm{~A}+750 \mathrm{D}$, the $u v$ range covered at $5.5 \mathrm{GHz}$ by the $2-\mathrm{GHz}$ bandwidth is $(0.5,129) \mathrm{k} \lambda$ (Table 1$)$. At $9.0 \mathrm{GHz}$, the baseline

\footnotetext{
4 The number of expected extragalactic sources in the images presented here can be estimated. Following either Anglada et al. (1998) or, independently, the results previously obtained by Purcell et al. (2010) of the CORNISH project, the expected extragalactic background objects, above $5 \sigma(=3 \mathrm{mJy})$ in a $\left(10^{\prime}\right)^{2}(=0.03 \mathrm{sq} \mathrm{deg})$ field area, at the $5.5-\mathrm{GHz}$ band, is close to unity.
} 
Table 2. Parameters of discrete $5.5 \mathrm{GHz}$ radio sources detected toward RCW 49 .

\begin{tabular}{|c|c|c|c|c|c|c|}
\hline Id & $\begin{array}{l}\text { RA, Dec (J2000) } \\
\text { (hms, dms) }\end{array}$ & $\begin{array}{l}\text { Total } S_{5.5 \mathrm{GHz}} \\
\quad(\mathrm{mJy})\end{array}$ & $\begin{array}{c}\text { Peak } S_{5.5 \mathrm{GHz}} \\
(\mathrm{mJy} / \text { beam })\end{array}$ & Nearest cataloged source & $\begin{array}{l}D \\
(\operatorname{arcsec})\end{array}$ & Comments \\
\hline $\mathrm{a}$ & $10: 25: 20.40,-57: 40: 43.3$ & $12 \pm 2$ & $3.4 \pm 0.6$ & IRAS 10236-5723 & 115 & \\
\hline \multirow[t]{3}{*}{$\mathrm{b}$} & $10: 23: 17.70,-57: 36: 12.4$ & $23 \pm 2$ & $1.8 \pm 0.1$ & 2E $1021.5-5720$ & 50 & $X$-ray source ${ }^{1}$ \\
\hline & & & & 1RXS J102322.2-573548 & 48 & ROSAT source $^{2}$ \\
\hline & & & & CXO J102323.3-573749 & 108 & Chandra source \\
\hline $\mathrm{c}$ & $10: 22: 28.36,-57: 35: 30.9$ & $3.5 \pm 0.5$ & $1.6 \pm 0.1$ & TYC 8608-1017-1 & 62 & Star \\
\hline dd 1 & $10: 24: 54.61,-57: 48: 42.9$ & $12 \pm 1$ & $10.7 \pm 0.2$ & CXOU J102454.5-574842 & 0.24 & YSO candidate ${ }^{4}$ \\
\hline $\mathrm{dd} 2$ & $10: 24: 53.89,-57: 48: 52.2$ & $14 \pm 2$ & $10 \pm 0.8$ & CXOU J102454.5-574842 & 12 & YSO candidate ${ }^{4}$ \\
\hline e & $10: 22: 53.82,-57: 50: 46.1$ & $7 \pm 1$ & $3.2 \pm 0.4$ & TYC 8608-1463-1 & 40 & Star \\
\hline $\mathrm{f}$ & $10: 22: 33.58,-57: 56: 20.9$ & $15 \pm 2$ & $15.3 \pm 0.8$ & TYC 8608-366-1 & 21 & Star \\
\hline
\end{tabular}

Notes. ${ }^{(1)}$ Belloni \& Mereghetti (1994); (2) Voges et al. (2000); ${ }^{(3)}$ Evans et al. (2010); ${ }^{(4)}$ Tsujimoto et al. (2007).
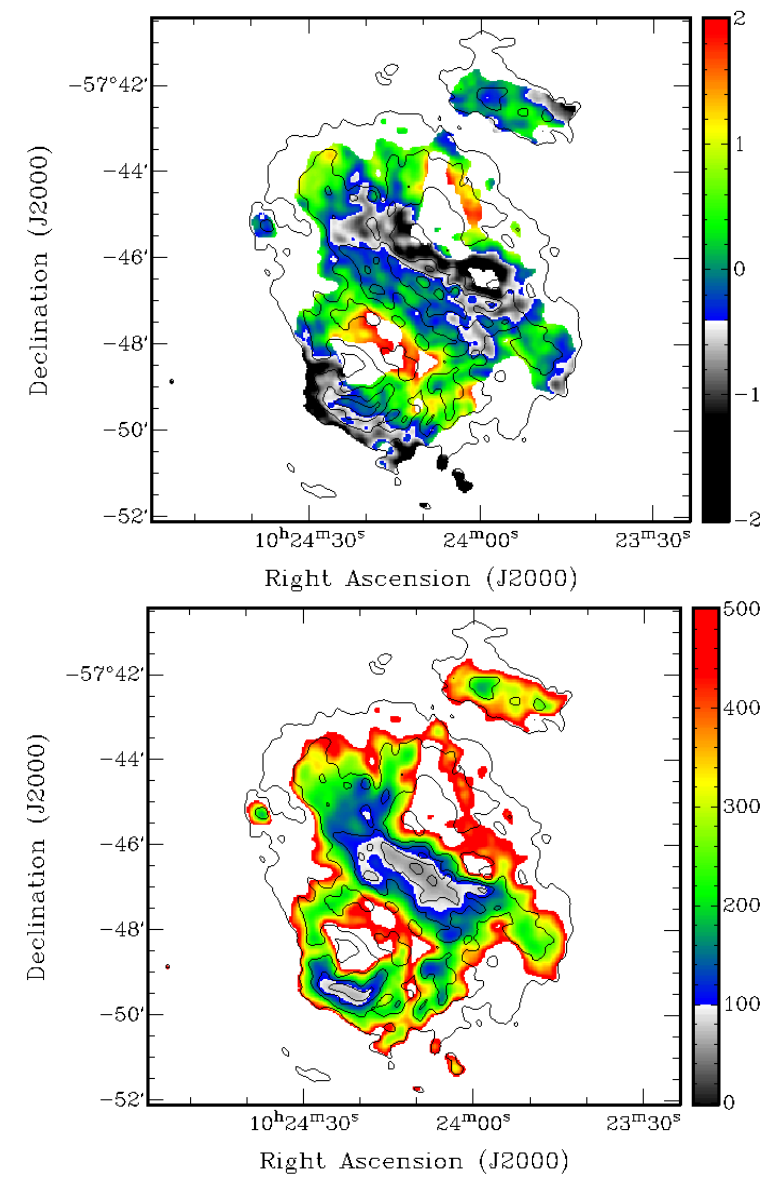

Fig. 5. Top: spectral index distribution image using the WU97 1.4-GHz and 2.4-GHz data at baselines $\geq 0.25 \mathrm{k} \lambda$. This value corresponds to angular scales of $\sim 25^{\prime}$. The black contours represent continuum emission at $2.4 \mathrm{GHz}$. Bottom: spectral index error distribution for the spectral index values above, in thousandths.

range is $(0.8,198) \mathrm{k} \lambda$. We built spectral index images using the same minimum baseline value of $\sim 1 \mathrm{k} \lambda$. Figure 6 shows the resulting spectral index map and the spectral index error distribution.

The bridge is the only part of the region that has sufficient signal for spectral index values to be determined. As in the 1.4 and $2.4 \mathrm{GHz}$ index map, the bulk of the emission in the bridge has index values $\sim 0$, but there appear to be some areas, associated with the brightest emission, that have values $\sim-0.4$, with low uncertainty.
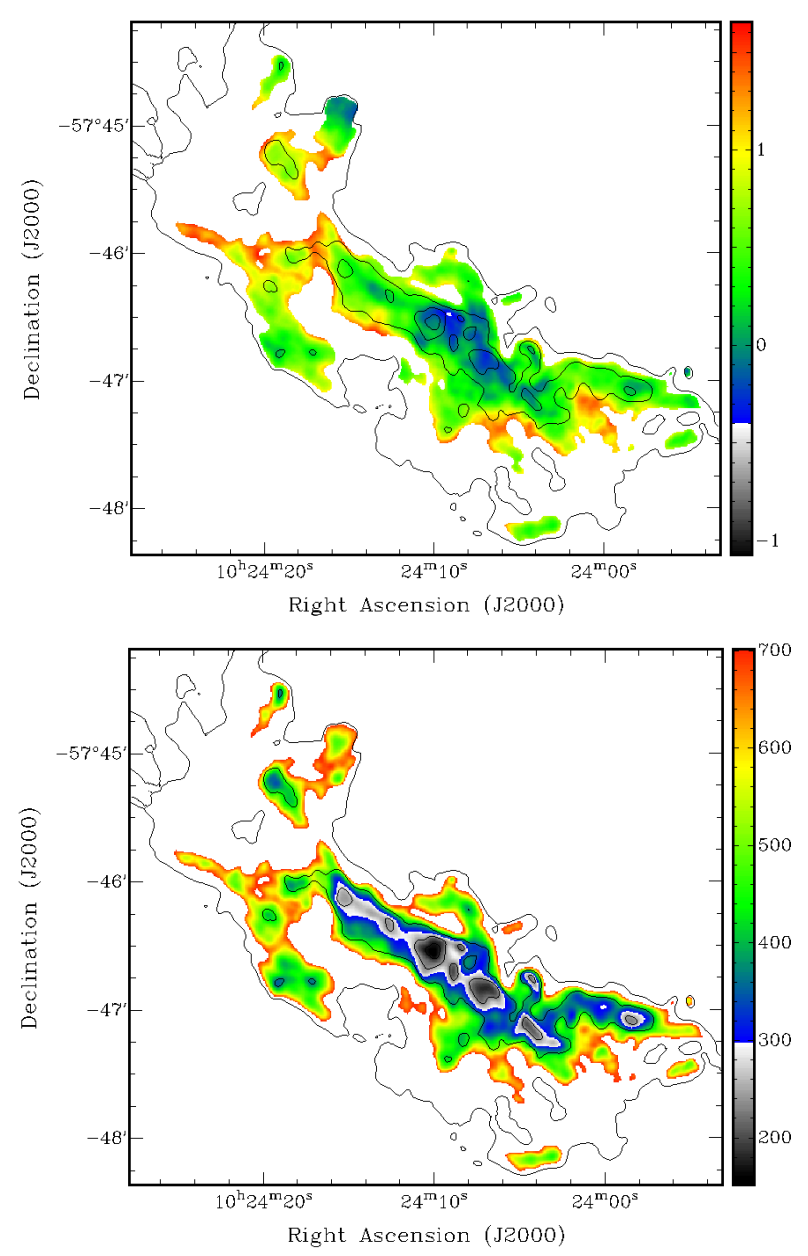

Fig. 6. Top: spectral index distribution based on 5.5 and $9.0-\mathrm{GHz}$ for baselines $\geq 1 \mathrm{k} \lambda$. Contours represent the $9.0-\mathrm{GHz}$ continuum data. Bottom: spectral index error distribution image, in thousandths.

The 2-GHz bandwidth at $5.5 \mathrm{GHz}$. The large 2-GHz extent of the CABB bandwidth allowed us to try the experiment of dividing the $5.5 \mathrm{GHz}$ data into two data sets, with central frequencies of 5.02 and $5.94 \mathrm{GHz}$, both with a bandwidth of $460 \mathrm{MHz}$. The continuum images at 5.02 and $5.94 \mathrm{GHz}$ revealed different flux levels. However, the spectral index map built using these data had large uncertainties due to the frequency proximity of the two data sets, and we abandoned any idea of interpreting the data. 


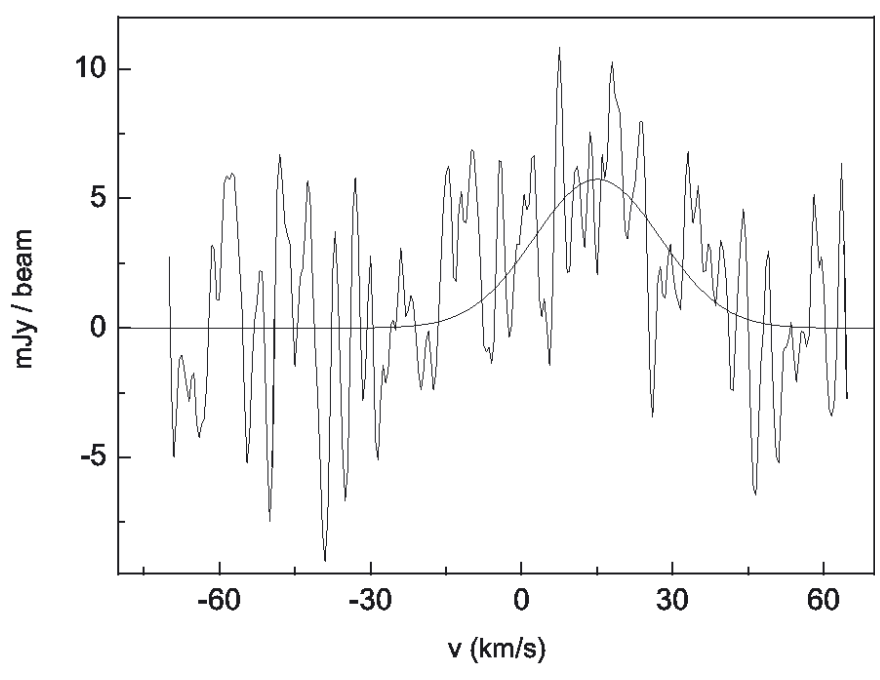

Fig. 7. Spectrum of the RRL H137 $\beta$ line near $5005 \mathrm{MHz}$, averaged over the maxima of the $5.5 \mathrm{GHz}$ ATCA continuum image $\left(S_{\text {cont }} \geq\right.$ $30 \mathrm{mJy}$ beam $^{-1}$ ), and Gaussian fit with a peak flux of $6 \mathrm{mJy}^{\text {beam }}{ }^{-1}$, a central velocity (LSR) of $+15 \mathrm{~km} \mathrm{~s}^{-1}$, and a velocity width of $25 \mathrm{~km} \mathrm{~s}^{-1}$.

\subsection{Line results}

Figure 7 shows the spectrum at $5.005 \mathrm{GHz}$ averaged over the continuum emission bridge region, over pixels with $S>$ $30 \mathrm{mJy}$ beam $^{-1}$ in the radio continuum. There is emission detected above the noise level at the frequency of the RRL H137 $\beta$ transition.

The expected rms that corresponds to the instrumental settings we used ${ }^{5}$ is $1.8 \mathrm{mJybeam}^{-1}$. The measured rms is $2 \mathrm{mJybeam}^{-1}$. The detected line has a peak flux density of $\sim 6 \mathrm{mJy}$ at a center velocity of $15 \mathrm{~km} \mathrm{~s}^{-1}$ and a velocity width of $25 \mathrm{~km} \mathrm{~s}^{-1}$. The continuum flux density, averaged in the same region, is $S_{\mathrm{c}}=2.5 \mathrm{Jy}$.

Caswell \& Haynes (1987) listed RRL emission from RCW 49 at the $\mathrm{H} 109 \alpha$ line with a central velocity of $0 \mathrm{~km} \mathrm{~s}^{-1}$ and a velocity width of $46 \mathrm{~km} \mathrm{~s}^{-1}$. Churchwell et al. (1974) also detected $\mathrm{He} 109 \alpha$, with a central velocity of $-4 \pm 1 \mathrm{~km} \mathrm{~s}^{-1}$, and a velocity width of about $50 \mathrm{~km} \mathrm{~s}^{-1}$ (data collected with the singledish Parkes telescope).

In search for anomalous microwave emission on small angular scales of the RCW 49 core, Paladini et al. (2013) observed the bridge region at $\mathrm{H} 109 \alpha$ with the ATCA (in 2009). They drew spectra at four positions along the bridge and detected the line at three of them. Their measured central velocities correspond to $14.5 \pm 1.8,-4.1 \pm 5.5$, and $14.5 \pm 1.8 \mathrm{~km} \mathrm{~s}^{-1}$, in full agreement with the parameters found here for $\mathrm{H} 137 \beta$. These velocities are also consistent with those of the highest velocity molecular cloud reported by Furukawa et al. (2009).

\subsection{Stellar bow shocks}

Using Spitzer-GLIMPSE data, Povich et al. (2008) discovered three stellar bow shocks in the region of RCW 49 and named them RCW 49-S1, -S2, and -S3. The authors proposed that the regions are physically related to RCW 49 and that $\mathrm{S} 2$ and S3 were still inside the HII region. They suggested that S1 may be formed by the combined action of the stellar winds of

\footnotetext{
5 http://www.narrabri.atnfcsiro.au/myatca/sensitivity_ calculator.html
}

Westerlund 2 stars that have escaped from the HII region at hundreds of $\mathrm{km} \mathrm{s}^{-1}$.

In addition to the detection at the GLIMPSE bands, RCW 49-S1 is a point source at MSX and IRAS images. The exciting star would be an $\mathrm{O} 5 \mathrm{III}$ if the distance to the complex is $6.1 \mathrm{kpc}$ (Povich et al. 2008). The spectral energy distribution of source S1 has been modeled by Povich et al. (2008, see their Fig. 3: Top). To match the 60 and $100 \mu \mathrm{m}$ IRAS fluxes the authors assumed the presence of low-density material farther from the star. They produced a model of the emission from a shell that is $2-3$ pc from the star. Their model predicts a shell flux of $\sim 0.15 \mathrm{Jy}$ at $1000 \mu \mathrm{m}$.

We searched for ATCA-5.5 GHz continuum sources at the positions of the bow shocks S1, S2, and S3. The images revealed emission consistent with bow-shock features positionally coincident with RCW 49-S1 and -S3. Even if $\mathrm{S} 2$ radiates in radio, intense extended $5.5 \mathrm{GHz}$ emission present at the position of S2 will be hiding the feature from view.

Figure 8 shows the $5.5 \mathrm{GHz}$ continuum emission at the locations of S1 and S3. The total flux densities are $70 \pm 10 \mathrm{mJy}$ and $140 \pm 20 \mathrm{mJy}$, respectively. There is some emission at the position of $\mathrm{S} 3$ at $9 \mathrm{GHz}$ but it is below $3 \sigma . \mathrm{S} 1$ is off the $9-\mathrm{GHz}$ continuum mosaic image.

Assuming that the gas forming the radio source coincident with $\mathrm{S} 1$ is optically thin $(\alpha \sim 0)$, the expected flux at $1000 \mu \mathrm{m}$ is $S_{1000 \mu \mathrm{m}} \approx 0.07 \mathrm{mJy}$. If the $5.5 \mathrm{GHz}$ source is a bow shock, one can use the $S_{1000 \mu \mathrm{m}}$ value as a key parameter to distinguish between different models of envelopes developed as in Povich et al. (2008).

\subsection{Surroundings of star MSP 18}

To the north of the region of Westerlund 2 we have discovered a jet-like structure at both radio bands (Fig. 9), of $\sim 20^{\prime \prime}$ $(0.01 \mathrm{pc}$ if the distance is $6 \mathrm{kpc})$. There are a number of known sources closely associated with the position of the jet, including two YSO candidates, a pre-main-sequence star, and the bright $\mathrm{O}$-supergiant MSP \#18. This last object is a Westerlund 2 member, marginally detected in the $5.5 \mathrm{GHz}$ full angular resolution image. The peak flux is $3.1 \mathrm{mJybeam}^{-1}$, and the background level at its position is $\sim 2.5 \mathrm{mJy}^{-1}$ beam ${ }^{-1}$ (see Sect. 5.1 for a discussion).

It is not clear if any one of the objects mentioned before is the source of the jet. The jet-like structure is surrounded by filaments at both sides, which appear to be forming a shell or bubble.

We estimated the spectral index value of the brightest part of the jet to be $-2.2 \pm 0.3$, based on an average taken over the pixels where the spectral index error was below or equal to 0.3. Such a negative spectral index denotes the conjunction of a steep injection spetrum in addition to very strong cooling $\left(\propto E^{2}\right.$, Vila \& Aharonian 2009, 24).

The jet-like source is also detected in the $8 \mu \mathrm{m}$-IRAC band (Fig. 9, bottom). An investigation of the nature of the jet-like structure, its possible physical association to the bubble, energetics, and the relation with other sources in the field is under way and will be reported elsewhere.

\section{Radio, IR, and X-ray emission from RCW 49}

Figure 10 portrays the emission at radio and near-IR, showing the free-free emission from the ionized gas and dust (polycyclic aromatic hydrocarbons, PAH) emission. The stars from the Westerlund 2 cluster are detected at IRAC band 1. There 

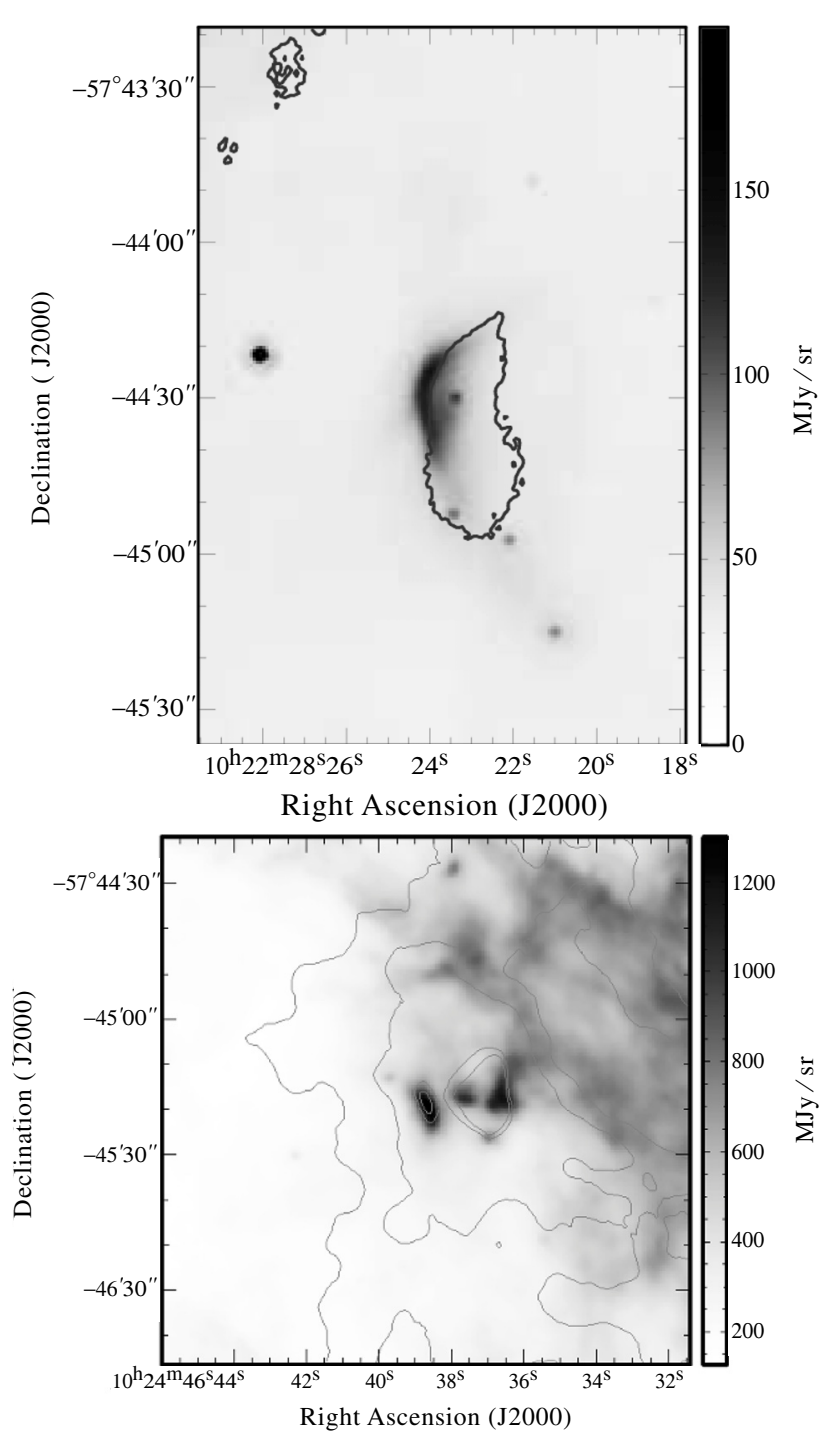

Fig. 8. Top: ATCA $5.5 \mathrm{GHz}$ continuum emission contours toward the object S1 from Povich et al. (2008). Levels are at 1.8 and $3 \mathrm{mJy}_{\text {beam }}{ }^{-1}$. In grayscale: emission at $8 \mu \mathrm{m}$ from Spitzer-IRAC. Bottom: $5.5-\mathrm{GHz}$ emission toward the object S3 from Povich et al. (2008) at the center of the image. Levels are at $3,5,11$, and $12 \mathrm{mJy}$ beam $^{-1}$. In grayscale: emission at $8 \mu \mathrm{m}$ from Spitzer-IRAC.

is some intense band 4 emission $(8 \mu \mathrm{m})$ in the bridge region. At the frequency of IRAC-band 4 the emission from PAHs is higher than at other bands (see Reich et al. 2006, their Fig. 1). Bright emission accounts for a dust-rich region, with the consequent effect of radiation reddening. IRAC band 4 also shows a discrete source at the position of the star MSP \#18, near the jet-like source described above.

The structures revealed in the radio are very similar to those observed in the near-IR by GLIMPSE. The northern and southern shells, and the bridge described by WU97 are very evident. It is clear that the HII region is not in an area of uniform density, with a lack of near-IR emission on the west side of RCW 49 suggesting lower material density to the west. This explains the possible source of the radio "blister" structure on the west side of the RCW 49 HII region mentioned by WU97.

We correlated the $5.5-\mathrm{GHz}$ continuum data with Chandra data taken by Nazé et al. (2008) and found no apparent associations.
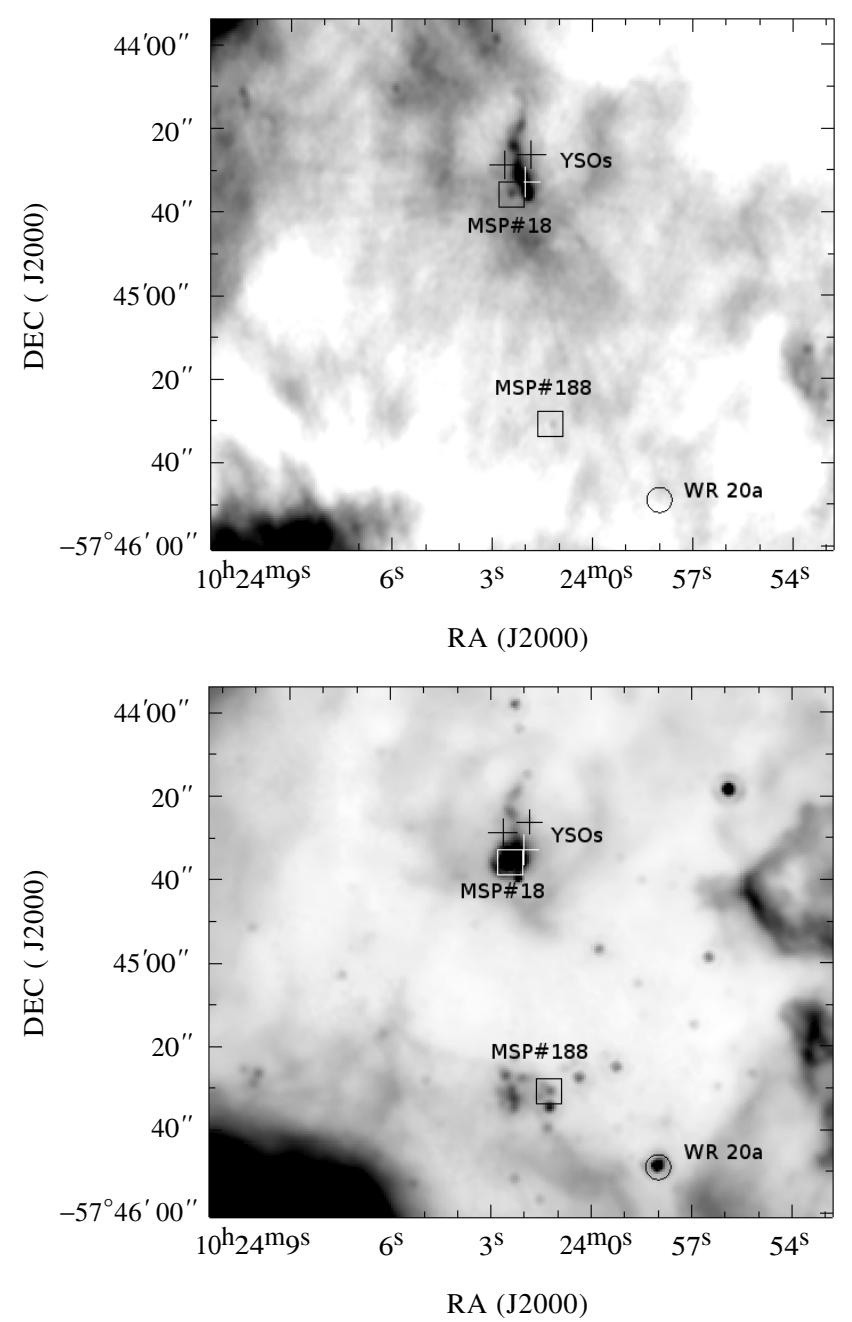

Fig. 9. Top: radio emission at $5.5 \mathrm{GHz}$ showing a jet-like source. Bottom: the same region at the $8 \mu \mathrm{m}$-IRAC band. Surrounding the jetlike source appears a closely circular ring of emission. The symbols represent the positions of known YSO candidates (crosses) and the Westerlund 2 stars MSP\#18, MSP\#188 (open squares), and WR 20a (circle).

The Fermi-pulsar region. We surveyed the region of the Fermi pulsar 2FGL J1022.7-5741, searching for a signature of a pulsar wind nebula. There is some emission at a level of $2 \mathrm{mJy}$, but it remains close to the noise. A higher-sensitivity observation is needed to image this emission and study weather it is associated with the pulsar.

\section{Discussion}

\subsection{Distance to RCW 49 and Westerlund 2}

The distance to Westerlund 2 and RCW 49 is somewhat uncertain owing to the large scatter in the values derived (see Table 3). The bulk of the distance estimates falls between 5 and $8 \mathrm{kpc}$, but there are some notable outliers. The radio observations presented here let us draw some general conclusions related to the distance discussion.

The HII region $R C W 49$. The central velocity of the putative $\mathrm{H} 137 \beta$ line detected in this study is similar to one of the CO line components found by Furukawa et al. (2009) and Ohama et al. (2010), to the "B" component in the spectrum shown by Dame (2007), and to the H109 $\alpha$ line recently found by Paladini et al. (2013). The central line velocity of $+15 \mathrm{~km} \mathrm{~s}^{-1}$ corresponds to a 


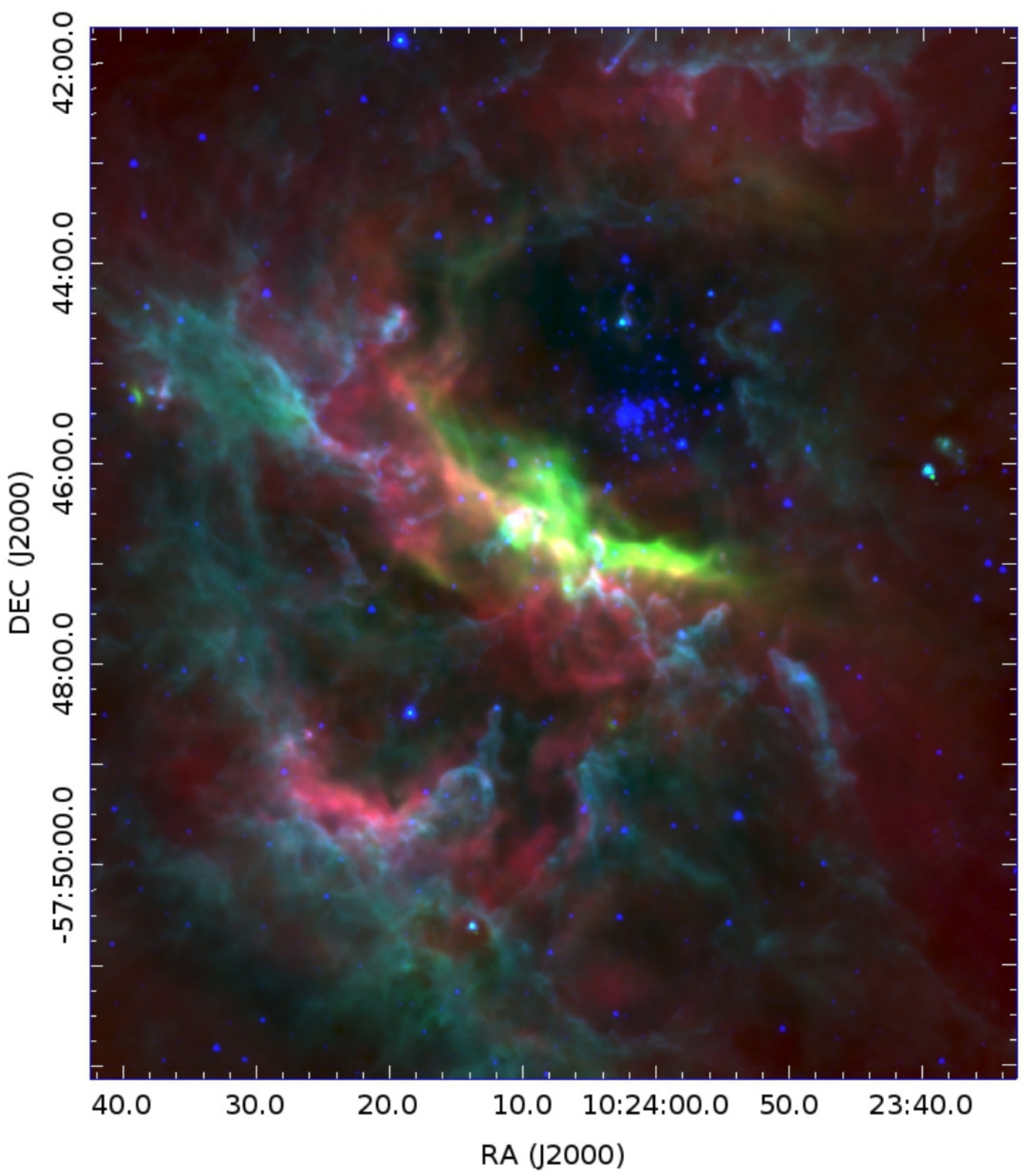

Fig. 10. RCW 49 field as seen at radio continuum ( $9 \mathrm{GHz}$, in red) and Spitzer-GLIMPSE band $1(3.6 \mu \mathrm{m})$ in blue and band $4(8 \mu \mathrm{m})$ in green. The stars in the field of view, including the core of the cluster, are clearly visible at $3.6 \mu \mathrm{m}$. The close association of the near-IR and radio emission is evident in the north and south shells and in the bridge.

kinematic distance of $\sim 6 \mathrm{kpc}$, according to the rotation curve in the outer galaxy derived by Brand \& Blitz (1993). The emitting ionized gas corresponds to the brightest continuum features that form the RCW 49 region. The fact that the ionized gas and part of the $\mathrm{CO}$ gas have similar velocities suggests that the ionized and the molecular components are physically associated.

The cluster Westerlund 2. Even if Westerlund 2 is located as close as $2.5 \mathrm{kpc}$, the thermal flux from the individual stellar winds of cluster members would not be detectable, well below the noise level of the images presented here. We estimate the expected $5.5 \mathrm{GHz}$ flux from the the late O-type supergiants in the cluster (e.g. Moffat et al. 1991) as $0.1 \mathrm{mJy}$ for a distance of $2.5 \mathrm{kpc}$, and as $0.01 \mathrm{mJy}$ for a distance of $6 \mathrm{kpc}$.

At the position of the Westerlund 2, the 5.5-GHz continuum emission shows diffuse emission at a level of $\sim 3 \mathrm{mJy}$.
No correlation between stellar positions and peaks in the radio flux were found, except for maxima in the radio emission of $1.6 \mathrm{mJybeam}^{-1}$ at the exact position of MSP 188, and of $3.1 \mathrm{mJybeam}^{-1}$ at the location of MSP 18. Given the $3.0 \mathrm{mJy}$ of diffuse emission, these are at best potential detections. Construction of images using only long baselines to eliminate the contribution of diffuse emission did not support detections of MSP 18 or 188, mainly because of the significantly smaller number of visibilities in the long-baseline range used and the commensurate rise in the rms level of the resulting image.

If we assume that the measured fluxes are due to thermal emission from the winds, we can estimate the corresponding mass loss rates. Assuming the spectral types of MSP 18 and MSP 188 to be O5 III and O4 III (Rauw et al. 2007) and wind terminal velocities from Prinja et al. (1990), the apparent 
Table 3. Summary of distance estimates for RCW 49/Westerlund 2.

\begin{tabular}{lclll}
\hline \hline Target & $d(\mathrm{kpc})$ & Band/Method & Component & Reference \\
\hline RCW 49 & 6 & Radio observations & Radio nebula & Westerlund (1961) \\
Wd2 & 5 & Optical photometry & Stars & Moffat \& Vogt (1975) \\
RCW 49 & 0 or 4.9 & Radio recombination lines & Radio nebula & Caswell \& Haynes (1987) \\
Wd2 & $7.9^{1.2}$ & Optical photometry & Stars & Moffat et al. (1991) \\
RCW 49 & 2.31 & Kinematic of HII regions & Ionized gas & Brand \& Blitz (1993) \\
Wd2 & $5.7 \pm 0.3$ & Optical spectroscopy & Stars & Piatti et al. (1998) \\
Wd2 & 2.8 & Near-infrared photometry & Stars & Ascenso et al. (2007) \\
Wd2 & $8 \pm 1.4$ & Optical spectrophotometry & Early-type stars & Rauw et al. (2007) \\
RCW 49 and Wd2 & $2-5$ & X-ray photometry & T Tauri stars & Tsujimoto et al. (2007) \\
Wd2, HESS J1023-575 & $6 \pm 1$ & CO emission, HI absorption lines & Molecular gas & Dame (2007) \\
RCW 49 & 6 & IR & Bow shocks & Povich et al. (2008) \\
RCW 49 & $5.4_{1.4}^{1.1}$ & CO emission & Molecular gas & Furukawa et al. (2009) \\
Pulsar 1023-5746 & 2.4 & $\gamma$-ray pulsar pseudo-distance & Pulsar & Saz Parkinson et al. (2010) \\
Wd2 & $6.5-9$ & Optical spectrophotometry & Eclipsing early-type & \\
& & & stars + WR20 a & Rauw et al. (2011) \\
Wd2 & 3.0 & Optical spectrophotometry/ & & \\
& & anomalous extinction law & Bright stars & Carraro et al. (2013) \\
Wd2 & $4.42 \pm 0.07$ & HST spectrophotometry & 26 O-type stars & Vargas Álvarez et al. (2013) \\
\hline
\end{tabular}

fluxes give mass loss rates of a few $\times 10^{-4} M_{\odot} \mathrm{yr}^{-1}$ for the two stars, assuming a distance of 4-6 kpc. These are about two orders of magnitude higher than the expected $\sim 10^{-6} M_{\odot} \mathrm{yr}^{-1}$ from standard models (e.g. Vink et al. 2000).

Vargas Álvarez et al. (2013) found that MSP 188 is an $\mathrm{O} 4 \mathrm{~V}+\mathrm{O} 9 \mathrm{~V}$ binary system. Nazé et al. (2008) concluded that MSP 18 is assumed to be an interacting binary. If the radio maxima at the positions of the stars are due to emission from these binary systems, it is possible that the flux is due to nonthermal emission arising from colliding-wind regions between the stars, as reported for instance by Chapman et al. (1999).

\subsection{Spectral indices: thermal vs. nonthermal emission}

As indicated in Sect. 3.2, the bulk of the emission in the region RCW 49 has a value $\sim 0$, consistent with the optically thin thermal plasma expected from a large HII region such as RCW 49. However, the spectral index maps in Figs. 5 and 6 hint that some of the plasma in the bridge region may have spectral index values that are significantly steeper than -0.1 , suggesting the presence of nonthermal emission due to relativistic electrons.

We remain cautious about this potential indication due to concerns arising from the different spatial frequency coverage at the two frequencies used to generate the spectral index maps. Though we have kept the minimum baselines fixed when deriving a spectral index map, not all baselines are covered by the observations at each frequency, from the shortest baselines to the longest ones. Certainly, the broad-band CABB data provide more complete baseline coverage at each frequency and a higher degree of spatial frequency overlap between the observing frequencies. However, a disparity in coverage at the two frequencies remains.

Ideally, stronger evidence for the presence of nonthermal emission is required, such as attempting to detect polarized emission in the areas of the bridge. This will be challenging because of the high density of the thermal plasma in this region which will Faraday-scatter any polarized emission, possibly to a level where it is no longer detectable.

\subsection{Nature of HESS J1023-575}

If nonthermal emission is present in the bridge, a simple explanation of the source HESS J1023-575 is possible. The bridge is very bright at IR wavelengths. Spitzer-GLIMPSE images at the four bands (b1, b2, b3, and b4) are direct evidence that there is a significant amount of heated dust (Churchwell et al. 2004; Whitney et al. 2004). This medium is a target for relativistic particles to produce high-energy radiation. The relativistic particles in the region could be produced by the collective action of the winds of the Westerlund 2 massive stars. The net effect is to increase the local average cosmic ray density. The bright IR emission of the bridge proves to be high-density ambient matter, which in turn will favor the interaction between nonrelativistic and relativistic particles, for instance, protons. Evantually, pions are produced from proton-proton interaction. The neutral pions decay, giving rise to gamma rays over an extended area (because the bridge is extended). This extended source could in principle be identified as HESS J1023-575, while secondary electrons would be responsible of the synchrotron radiation. Deeper observations to increase the signal-to-noise ratio at better matching beams are a fundamental tool for confirming or discarding the detection of nonthermal emission from synchrotron origin and for testing this hypothesis.

\section{Conclusions and future work}

The broad-band ATCA CABB observations reveal a detailed view of radio-emitting plasma in RCW 49. For the first time pillars of radio emission were detected in the HII region RCW 49. The radio emission is very similar in morphology to the Spitzer-GLIMPSE emission, indicating that the plasma generates free-free emission at both radio and IR wavelengths.

The brightest continuum radio knots in the bridge region have recombination line emission. We detected the $\mathrm{H} 137 \beta$ line at a $3 \sigma$ level. The $\mathrm{H} 137 \beta$ line central velocity agrees very well with that of CO (Furukawa et al. 2009). We suggest that the molecular gas and the ionized matter are co-located at a kinematic distance of $6 \pm 1 \mathrm{kpc}$. 
Spectral indices derived from the simultaneous twofrequency data showed some hints of nonthermal radiation. We proposed an explanation for the high-energy emission based on a proton-proton interaction scenario. We did not detect a pulsar wind nebula around 2FGL J1022.7-5741 above a threshold of $2 \mathrm{mJy}$.

A detailed study of the correlation between cm-radio data and high-resolution IR data (e.g. Herschel images) will allow a more detailed description of the morphology of the plasma and shed light on the mechanisms that excite the matter, especially the dust. Observations of RRLs, such as H109 $\alpha$, can provide information on the parameters of the ionized emitting gas, and also on its velocity field and consequently its kinematic distance. Additional continuum data at 5.5 and $9 \mathrm{GHz}$ will provide more sensitivity, which is required to attempt to detect members of the Westerlund 2 cluster.

Acknowledgements. We thank the anonymous referee for the careful reading of our manuscript and useful comments. P.B. wishes to thank people at ATNF that helped in various ways, for instance, Nathan Pope, Marc Wieringa, Robin Wark, Vanessa Moss, Jill Rathborne, Jim Caswell, Shari Breen, and also G.E. Romero. We are grateful to Yael Nazé, who provided the Chandra data fits images, and to Roberta Paladini, who provided the results of RRL data of RCW 49 in advance of publication. J.M. and J.R.S.S. acknowledge support for different aspects of this work by grants AYA2010-21782-C03-03 from the Spanish Government and Consejería de Economía, Innovación y Ciencia of Junta de Andalucía as research group FQM-322, as well as FEDER funds. P.B. acknowledges support from PICT 2007, 00848 (ANPCyT). This research has made use of NASA's Astrophysics Data System Bibliographic Services, of the SIMBAD database, operated at CDS, Strasbourg, France and of the NASA/IPAC Infrared Science Archive, which is operated by the Jet Propulsion Laboratory, California Institute of Technology, under contract with the National Aeronautics and Space Administration.

\section{References}

Abramowski, A., Acero, F., Aharonian, F., et al. 2011, A\&A, 525, 46 Aharonian, F., Akhperjanian, A. G., Bazer-Bachi, A. R., et al. 2007, A\&A, 467, 1065

Anglada, G., Villuendas, E., Estallela, R., et al. 1998, AJ, 116, 2953
Ascenso, J., Alves, J., Beletsky, Y., \& Lago, M. T. V. T. 2007, A\&A, 466, 137 Belloni, T., \& Mereghetti, S. 1994, A\&A, 286, 935

Benjamin, R. A., Churchwell, E., Babler, B. L., et al. 2003, PASP, 115, 953 Brand, J., \& Blitz, L. 1993, A\&A, 275, 67

Carraro, G., Turner, D., Majaess, D., \& Baume, G. 2013, A\&A, 555, 50

Caswell, J. L., \& Haynes, R. F. 1987, A\&A, 171, 261

Chapman, J. M., Leitherer, C., Koribalski, B., et al. 1999, ApJ, 518, 890

Churchwell, E., Mezger, P. G., \& Huchtmeier, W. 1974, A\&A, 32, 283

Churchwell, E., Whitney, B. A., Babler, B. L., et al. 2004, ApJS, 154, 322

Dame, T. M. 2007, ApJ, 665, L163

Dormody, M. for the Fermi-LAT Collaboration 2009, Fermi Symposium Conf.

Proc. C091122 [arXiv: 0912.3949]

Evans, I. N., Primini, F. A., Glotfelty, K. J., et al. 2010, ApJS, 189, 37

Fujita, Y., Hayashida, K., Takahashi, H., \& Takahara, F. 2009, PASJ, 61, 1229

Furukawa, N., Dawson, J. R., Ohama, A., et al. 2009, ApJ, 696, 115

Goss, W. M., \& Shaver, P. A. 1970, Austr. J. Phys. Supp., 14, 1

Johnson, K. E. 2005, IAUS, 227, 413

Moffat, A. F. J., \& Vogt, N. 1975, A\&AS, 20, 155

Moffat, A. F. J., Shara, M. M., \& Potter, M. 1991, AJ, 102, 642

Nazé, Y., Rauw, G., \& Manfroid, J. 2008, A\&A, 483, 543

Nolan, M. C., Magri, C., Benner, L. A. M., et al. 2012, ApJS, 199, 46

Ohama, A., Dawson, J. R., Furukawa, N., et al. 2010, ApJ, 709, 975

Paladini, R., et al. 2013, ApJ, submitted

Piatti, A. E., Bica, E., \& Clariá, J. J. 1998, A\&AS, 127, 423

Povich, M. S., Benjamin, R. A., Whitney, B. A., et al. 2008, ApJ, 689, 242

Purcell, C. R., Hoare, M. G., \& Melvin, G. 2010, Highlights of Astronomy, ed. I. F. Corbett, 15 (Cambridge: University Press)

Rauw, G., Manfroid, J., Gosset, E., et al. 2007, A\&A, 436, 981

Rauw, G., Sana, H., \& Nazé, Y. 2011, A\&A, 535, 40

Reich, W. T., Rho, J., Tappe, A., et al. 2006, AJ, 131, 1479

Robinson, B. J., Brooks, J. W., Godfrey, P. D., \& Brown, R. D. 1974, Austr. J. Phys., 27, 856

Rodgers, A. W., Campbell, C. T., \& Whiteoak, J. B. 1960, MNRAS, 121, 103

Sault, R. J., Teuben, P. J., \& Wright, M. C. H. 1995, ASPC, 77, 433

Saz Parkinson, P. M., Dormody, M., Ziegler, M., et al. 2010, ApJ, 725, 571

Tsujimoto, M., Feigelson, E. D., Townsley, L. K., et al. 2007, ApJ, 665, 719

Vila, G. S., \& Aharonian, F. 2009, in Compact Objects and their Emission, eds. G. E. Romero, \& P. Benaglia, AAABS, 1, 1

Vink, J. S., de Koter, A., \& Lamers, H. J. G. L. M. 2000, A\&A, 362, 295

Voges, W., Aschenbach, B., Boller, Th., et al. ROSAT Faint source Cat. 2000, IAU Circ., 7432, 1

Westerlund, B. 1961, ArA, 2, 419

Whiteoak, J. B. Z. \& Uchida, K. I. 1997, A\&A, 317, 536

Whitney, B. A., Indebetouw, R., Babler, B., L. et al. 2004, ApJS, 154, 315

Wilson, W. E., Ferris, R. H., Axtens, P., et al. 2011, MNRAS, 416, 832 